
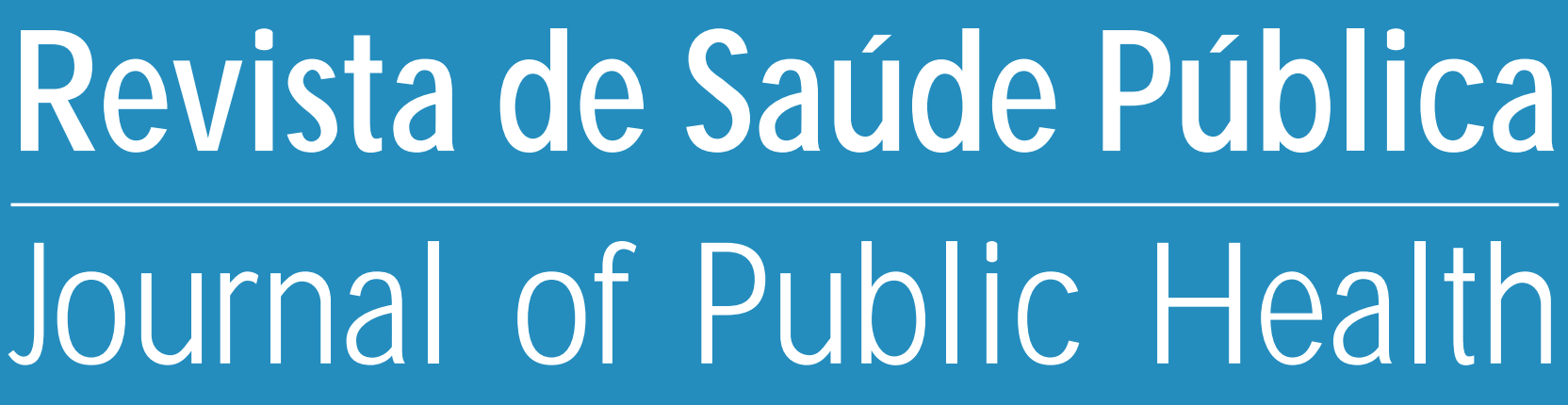

\title{
Espécies de Anopheles (Culicidae, Anophelinae) em área endêmica de malária, Maranhão, Brasil
}

\section{Species of Anopheles (Culicidae, Anophelinae) in a malaria-endemic area, Maranhão, Brazil}

Maria M dos SP Xavier e José M M Rebêlo N úcleo de Patologia Tropical e Medicina Social da Universidade Federal do Maranhão. São Luís, MA, Brasil 


\title{
Espécies de Anopheles (Culicidae, Anophelinae) em área endêmica de malária, Maranhão, Brasil
}

\section{Species of Anopheles (Culicidae, Anophelinae) in a malaria-endemic area, Maranhão, Brazil}

\author{
Maria M dos SP Xavier e José MM Rebêlo \\ Núcleo de Patologia Tropical e Medicina Social da Universidade Federal do Maranhão. São Luís, MA, \\ Brasil
}

\section{Descritores}

Anopheles. Ecologia de vetores. Malária, transmissão.
Keywords

Anopheles. Ecology,

vectors. Malaria,

transmission.

\section{Resumo}

Objetivo

Estudar a flutuação sazonal, frequiência horária, abundância relativa e riqueza de espécies de Anopheles em ambiente antrópico, para entender a bioecologia do grupo e para a monitorização do programa de controle da malária.

\section{Métodos}

Os anofelinos foram estudados durante um ano, de outubro de 1996 a setembro de 1997, das 18 às 6 horas, a cada 30 dias, no Município da Raposa, Ilha de São Luís, Maranhão. Utilizou-se o método de captura de fêmeas em iscas humanas no intra e peridomicílio, com tubo de sucção e foco luminoso orientado.

\section{Resultados}

Foram coletados 1.407 espécimes assim distribuídos: Anopheles aquasalis (82\%), Anopheles galvaoi $(10,2 \%)$ e Anopheles albitarsis (6,4\%). As demais espécies, Anopheles evansae, Anopheles nuneztovari $\mathrm{e}$ Anopheles triannulatus davisi representaram juntas 1,4\%. Os anofelinos ocorreram o ano inteiro, principalmente no período chuvoso, sendo mais freqüentes no intra $(75,3 \%)$ do que no peridomicílio $(24,7 \%)$, preferindo sugar sangue no crepúsculo vespertino e nas primeiras horas da noite.

\section{Conclusão}

As variações observadas no comportamento do anofelino mostram que as diferentes espécies vêm adaptando-se, em maior ou menor grau, ao convívio com o homem nas suas habitações.

\begin{abstract}
Introduction

The study of the seasonal fluctuation, nocturnal activity, relative abundance and the richness of Anopheles species in anthropic environment is essential to the understanding of the their bioecology and to the surveillance program of malaria control. Methods

The Anopheles species were studied from 6 P.M. to 6 A.M., once a month, for one year, from October 1996 to September 1997, in the municipal district of Raposa, of the São Luís island, Maranhão state. The basic method was the capture of female specimens on human baits in peri and intradomicile sites by means of aspiration
\end{abstract}




\begin{abstract}
tube and guided luminous focus.
Results

A total of 1.407 specimens were collected and distributed as follow: Anopheles aquasalis ( $82 \%$ of the sample), Anopheles galvaoi (10,2\%), Anopheles albitarsis (6,4\%), Anopheles evansae, Anopheles nuneztovari, and Anopheles triannulatus davisi (the last three represented together $1,4 \%$ ). The anophelines occurred all year round, mainly in the rainy period, being more frequent in the intra $(75,3 \%)$ than in the peridomicile site $(24,7 \%)$, showing a clear preference to suck blood in the evening.

Conclusion

The behavioural variation of Anopheles shows that the different species are becoming adapted to closeness to human habitations, in the rural zone of the São Luis island.
\end{abstract}

\section{INTRODUÇÃO}

A malária, a mais expressiva endemia do Estado do Maranhão, tem sido um fenômeno constante apesar dos esforços da FNS (Fundação Nacional de Saúde) e de sua contribuição para diminuir e até mesmo controlar a transmissão em algumas áreas através de borrifação de inseticida no intra (Cipermetrina) e no peridomicílio (Malathion), da procura dos doentes, do diagnóstico e do tratamento.

Os casos de malária registrados na Ilha de São Luís, em sua maioria, são importados da Pré-Amazônia e da Baixada, consideradas as áreas malarígenas do Estado. Essas áreas apresentam características climáticas e geográficas propícias, expressiva densidade anofélica (Rebêlo et al..$^{15}$, 1997), fluxo migratório intenso e constante exposição do homem ao vetor em decorrência de suas atividades diárias, tais como: pesca como meio de subsistência, extração de madeira, preparo da terra para pastagens e lavoura, entre outros.

No Maranhão, a existência de áreas de alta transmissão de malária vem-se constituindo em importante problema de saúde pública, exercendo fortes pressões nas áreas vizinhas. Dentro deste contexto, a Ilha de São Luís, situada na zona do golfão maranhense, tem sofrido sérias consequiências, pois tem sido alvo constante do fluxo migratório das populações interioranas. Este fenômeno vem causando a ampliação das áreas urbanas e, conseqüentemente, produzindo alterações nos habitats naturais dos vetores, gerando surtos epidêmicos.

Em 1996, foram confirmados 450 casos de malária na Ilha de São Luís, que abrange os municípios de São Luís, São José de Ribamar, Paço do Lumiar e o recentemente criado município da Raposa, alvo deste trabalho, o qual até setembro de 1997 já apresentava 117 casos de malária (Ministério da Saúde ${ }^{13}, 1997$ ). No ano de 1997 foram 1.283 casos registrados na Ilha de São Luís, sendo que 1.253 eram por Plasmodium vivax. Em 1998, o número de lâminas examinadas positivas foi de 1.150 , dos quais 1.086 eram Plasmodium vivax $\left(\mathrm{Silva}^{17}, 1999\right)$.

Apesar da incômoda situação do Maranhão em relação à malária, conhece-se muito pouco sobre a dinâmica vetorial das espécies de anofelinos que ocorrem no Estado. Até o momento, tem-se conhecimento apenas do levantamento realizado na Amazônia maranhense por Rebêlo et al. ${ }^{15}(1997)$. Esses autores estudaram a fauna de anofelinos de Buriticupu e detectaram a presença dominante de Anopheles darlingi, inclusive dentro dos domicílios, e o incriminaram como o principal vetor da malária humana naquela região.

No presente trabalho estudam-se os anofelinos em área domiciliar, caracterizando a flutuação sazonal, a freqüência horária, a riqueza e a abundância relativa das espécies que se associam com o homem, em área malarígena do município da Raposa. O que se pretende é ampliar os conhecimentos sobre estes importantes vetores no Maranhão e indicar meios que possam ajudar os órgãos de saúde e as comunidades no sentido de minimizar o contato homem-vetor e desta forma controlar o avanço da malária no Estado.

\section{MÉTODOS}

\section{Área de Estudo}

A Ilha de São Luís localiza-se no litoral setentrional brasileiro, a $2^{\circ} 32^{\prime} \mathrm{LS}$ e $44^{\circ} 43^{\prime} \mathrm{LW}$. Limita-se ao norte e a leste com o oceano Atlântico, a oeste com a baía de São Marcos, a leste com a baía de São José e ao sul com a baía do Arraial e estreito dos Mosquitos, que a separa do continente. O litoral é bastante recortado, com largos estuários e relevos residuais de pequena altitude, constituídos por terrenos baixos, elevandose a $32 \mathrm{~m}$ de altitude onde está localizada São Luís, capital do Estado do Maranhão (IBGE ${ }^{8}$, 1984). A Ilha tem uma área de $905 \mathrm{~km}^{2}$, sendo politicamente dividida em 4 municípios: São Luís, São José de Ribamar, Paço do Lumiar e Raposa, recentemente emancipado. 
A rede hidrográfica é representada por diversos rios e estuários que cortam a ilha, sendo muitos deles influenciados pelas marés, entre eles os rios Anil, Bacanga, Tibiri e Mosquito. Entre os pequenos rios pode-se citar Mocajituba, Cururuca, Antônio Neves, São João e Paciência. Existem numerosos córregos, igarapés e riachos que concorrem para a irrigação natural do solo da Ilha. (IBGE $\left.{ }^{8}, 1984\right)$.

Até algum tempo atrás, a vegetação era um misto da floresta latifoliada e babaçual (Attalaea phalerata), além da floresta paludosa marítima (manguezal). Ultimamente, a ecologia da Ilha vem-se modificando em função da demanda imobiliária e retirada constante e desenfreada da cobertura vegetal. Os remanescentes da vegetação original são representados por coberturas florísticas secundárias e extensas áreas de capoeiras baixas com intrusões de babaçu, com manchas de cerrados e formações pioneiras (campo e restinga). Margeando os rios, onde ainda há matas ciliares, predominam os buritizais (Mauritia vinifera) e juçarais (Euterpe oleraceae) (IBGE $\left.{ }^{8}, 1984\right)$.

O clima é tropical mesotérmico e úmido, com duas estações bem definidas. A chuvosa, de janeiro a junho, concentra, em média, cerca de $94 \%$ do total anual das chuvas; a estação seca, de julho a dezembro, concentra apenas $6 \%$. O total pluviométrico é elevado, em torno de $1.900 \mathrm{~mm}$ anuais, em média. As temperaturas são elevadas durante o ano todo (média de $26^{\circ} \mathrm{C}$ ) com variação anual pequena $\left(\mathrm{IBGE}^{8}, 1984\right)$.

O presente estudo foi realizado na localidade de Primirim, no município da Raposa, situado a nordeste da ilha, limitando-se ao norte com o oceano Atlântico, ao sul com Paço do Lumiar, a leste com a baía de São José de Ribamar. Primirim está situada à margem esquerda da estrada que dá acesso à sede do município. Compreende área baixa de planície flúviomarinha atingida pelas oscilações das marés. A faixa litorânea é dominada por vegetação estacional perenifólia paludosa marítima, representada pelo manguezal. A zona de contato de terra firme é caracterizada pela presença de capoeira intermediada por áreas de dunas e restingas. No local de estudo predominam associações de solos alagados, salinos e mal drenados.

\section{Amostragem}

Os anofelinos foram coletados durante um ano (outubro/96 a setembro/97), uma vez por mês, das 18 às 6 h. O método utilizado foi a captura de fêmeas em iscas humanas, com tubo de sucção tipo Castro e foco luminoso orientado. Foram estudados o peri e o intradomicílios, sendo que em cada ambiente permanecia um coletor e um membro da equipe de trabalho, que serviam como iscas. A troca de coletores por ambiente ocorria à meia-noite.

Os espécimes capturados a cada hora eram acondicionados em frascos mortíferos à base de acetato de etila e transferidos para depósitos de polietileno devidamente etiquetados. Ao final da captura, todo material era conduzido ao laboratório e identificado por técnicos.

Utilizou-se teste estatístico não paramétrico (quiquadrado) para analisar as diferenças entre os anofelinos capturados nas estações seca e chuvosa. As diferenças foram consideradas significativas quando a probabilidade (p) do erro foi inferior a $5 \%$ $(\mathrm{p}<0,05)$. Foi realizado o teste de correlação para verificar a influência dos fatores climáticos na densidade dos anofelinos ao longo do ano.

\section{RESULTADOS}

\section{Números de Espécies e Abundância Relativa}

Foram coletados 1.407 espécimes do gênero Anopheles de seis espécies, todas pertencentes ao subgênero Nyssorhynchus, a saber: An. (Nys.) albitarsis Arribalzaga, 1878 (lato sensu), An. (Nys.) aquasalis Curry, 1932, An. (Nys.) galvaoi Causey, Deane e Deane, 1943, An. (Nys.) evansae Brèthes, 1926, An. (Nys.) nuneztovari Gabaldón, 1940 e An. (Nys.) triannulatus davisi Peterson e Shannon, 1927.

An. aquasalis foi a espécie mais freqüente na área de estudo, sendo representada por $82 \%$ da amostra total. Seguem na ordem de importância, An. galvaoi (10,2\%) e An. albitarsis (6,4\%). As demais espécies, An. evansae, An. nuneztovari e An. triannulatus davisi representaram juntas $1,4 \%$ da amostra total (Tabela 1 ).

Tabela 1 - Números de Anopheles capturados em iscas humanas no peri e intradomicílio, em Primirim, Município da Raposa, Ilha de São Luís-MA, de outubro/1996 a setembro/1997.

\begin{tabular}{lrrrr}
\hline & \multicolumn{3}{c}{ Domicílio } & \multicolumn{3}{c}{ Total } \\
\cline { 2 - 5 } Anopheles & Peri & Intra & Indivíduos & $(\%)$ \\
\hline aquasalis & 280 & 874 & 1.154 & 82,0 \\
albitarsis & 27 & 63 & 90 & 6,4 \\
galvaoi & 32 & 111 & 143 & 10,2 \\
evansae & 2 & 6 & 8 & 0,6 \\
nuneztovari & 3 & 2 & 5 & 0,3 \\
triannulatus & 4 & 3 & 7 & 0,5 \\
\hline Total & 348 & 1.059 & 1.407 & 100,0 \\
(\%) & 24,7 & 75,3 & 100,0 & \\
\hline
\end{tabular}

\section{Preferência por Ambientes}

As atrações dos anofelinos às iscas foram mais freqüentes no intradomicílio (75,3\% da amostra total) do que no peri $(24,7 \%)$. As freqüências das três espécies 
mais abundantes na área foram significativamente maiores no intradomicílio ( $\mathrm{p}<0,05)$ : An. aquasalis $(75,7 \%)$, An. galvaoi (77,6\%) e An. albitarsis (70,0\%). Não houve diferença significativa entre o intra e peridomicílio para as espécies An. evansae, An. nuneztovari e An. triannulatus davisi $(\mathrm{p}>0,05)$. A espécie mais freqüente tanto no peri quanto no intradomicílio foi An. aquasalis (Tabela 1).

\section{Horário de Atividade}

A Tabela 2 mostra a distribuição das espécies de acordo com o horário e o ambiente em que cada uma foi encontrada. Considerando todas as espécies juntas observa-se uma acentuada freqüência de indivíduos entre $18 \mathrm{~h}$ e $23 \mathrm{~h}$, mas principalmente no crepúsculo vespertino, entre $18 \mathrm{~h}$ e $19 \mathrm{~h}$. Porém, podese perceber claramente que este padrão foi influenciado por An. aquasalis, em função do seu amplo predomínio, sendo, inclusive, a única espécie encontrada das 18 às $6 \mathrm{~h}$, nos dois ambientes. Já An. galvaoi só esteve presente em todos os horários no intra domicílio; no ambiente peridomiciliar restringiu sua atividade às primeiras horas da noite. As demais espécies representadas por um número menor de indivíduos não apresentaram um padrão de atividade definido.

\section{Flutuação Sazonal}

Os anofelinos estiveram em atividade hematofágica o ano inteiro, com grande abundância de indivíduos na estação chuvosa, principalmente no início, em fevereiro (Tabela 3, Figura). Nesta estação ocorreram os maiores índices pluviométricos e de umidade e as menores médias mensais de temperatura. A menor freqüência de indivíduos atraídos às iscas humanas ocorreu em outubro, final da estação seca.

As espécies An. triannulatus, An. galvaoi, An. albitarsis e An. aquasalis foram encontradas nas duas estações, mas com maior frequiência na chuvosa (Tabela 3 ), sendo as três últimas responsáveis pelo pico de fevereiro devido à alta atividade hematofágica delas naquele mês, no qual também foram encontrados os únicos cinco exemplares de An. nuneztovari. Ao contrário das demais espécies o pico de An. triannulatus ocorreu no final do período chuvoso.

An. aquasalis foi a única espécie encontrada em todos os meses, com dois picos de atividade, um no início da estação chuvosa e o outro no final da estação seca. Comparando a variação dos parâmetros ambientais com a atividade mensal deste anofelino, ao longo do ano, observou-se que a umidade apresentou maior correlação $(\mathrm{R}=0,40)$ do que a temperatura $(R=0,25)$. Já a precipitação não apresentou correlação positiva $(\mathrm{R}=0,06)$.

Quando se comparou o número de indivíduos por estação, pelo teste do qui-quadrado verificou-se que a freqüência deles foi significativamente maior na estação chuvosa $(\mathrm{p}<0,05)$, quando o índice pluviométrico alcançou valor muito maior $(1160 \mathrm{~mm})$ do que na estação seca (160mm). An. albitarsis foi abundante nas duas estações, porém não apresentou preferência por nenhuma $(\mathrm{p}>0,05)$. An. evansae ocorreu exclusivamente no período chuvoso.

Tabela 2 - Freqüência horária dos Anopheles capturados em iscas humanas, em Primirim, Município da Raposa, Ilha de São Luís-MA, de outubro/1996 a setembro/1997.

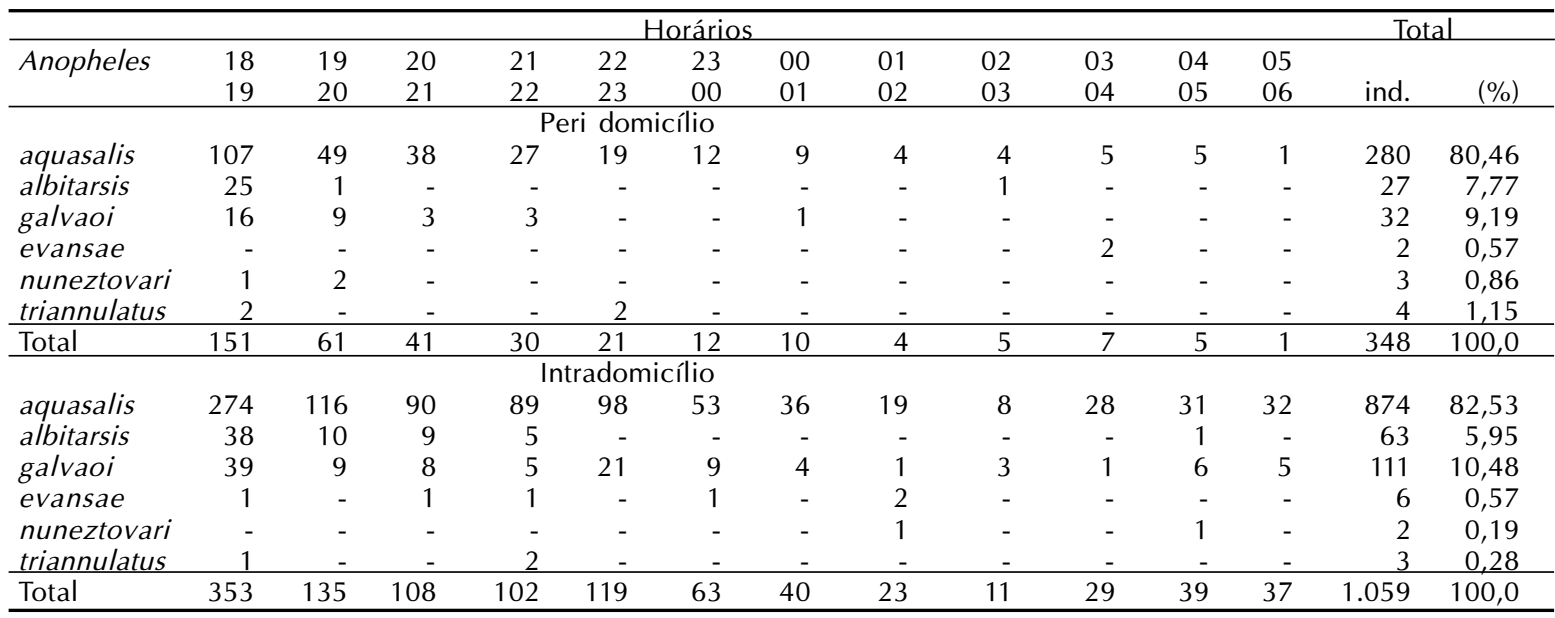


Tabela 3 - Números de Anopheles capturados mensalmente em iscas humanas no peri e intradomicílio, em Primirim, Município da Raposa, Ilha de São Luís-MA, de outubro/1996 a setembro/1997.

\begin{tabular}{|c|c|c|c|c|c|c|c|c|c|c|c|c|}
\hline \multirow{2}{*}{$\begin{array}{l}\text { Espécies de } \\
\text { Anopheles }\end{array}$} & \multicolumn{7}{|c|}{ Estação chuvosa } & \multicolumn{5}{|c|}{ Estação seca } \\
\hline & & $\mathrm{F}$ & $M$ & $\mathrm{~A}$ & $M$ & & & $\mathrm{~A}$ & $\mathrm{~S}$ & $\mathrm{O}$ & $\mathrm{N}$ & $\mathrm{D}$ \\
\hline aquasalis & 73 & 331 & 105 & 42 & 44 & 112 & 66 & 57 & 37 & 17 & 173 & 97 \\
\hline albitarsis & 2 & 31 & 5 & 1 & - & 3 & - & - & 5 & 10 & 21 & 12 \\
\hline galvaoi & - & 63 & 22 & 9 & 6 & 22 & 12 & 9 & - & - & - & - \\
\hline evansae & - & 4 & 3 & 1 & - & - & - & - & - & - & - & - \\
\hline nuneztovari & - & 5 & - & - & - & - & - & - & - & - & - & - \\
\hline triannulatus & - & - & - & 1 & 2 & 3 & 1 & - & - & - & - & - \\
\hline Total & 75 & 434 & 135 & 54 & 52 & 140 & 79 & 66 & 42 & 27 & 194 & 109 \\
\hline
\end{tabular}
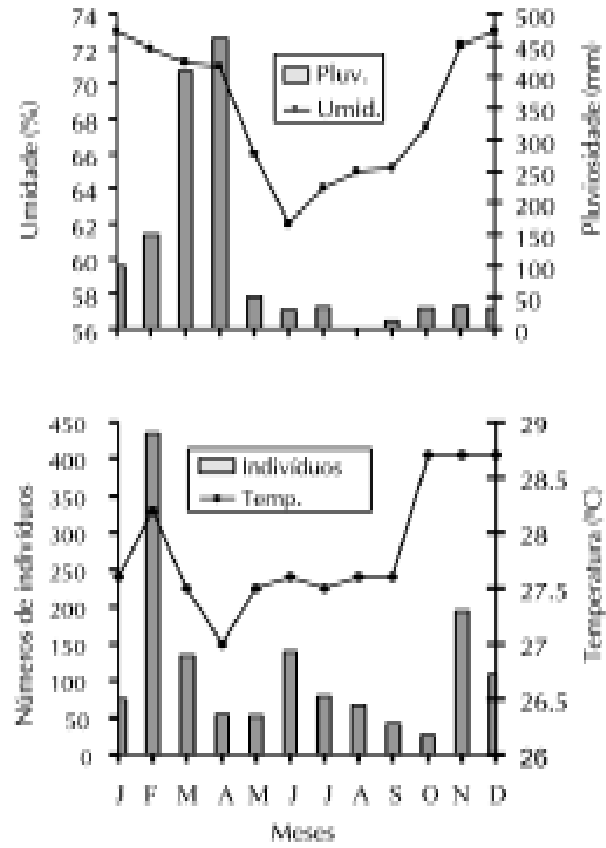

Figura - Variação mensal dos índices de pluviosidade $(\mathrm{mm})$, umidade relativa do ar $(\%)$, temperatura $\left({ }^{\circ} \mathrm{C}\right)$ e do número de espécimes de anofelinos coletados de outubro de 1996 a setembro de 1997, em Primirim, Município da Raposa, Ilha de São Luís, Maranhão.

\section{DISCUSSÃO}

Rebêlo et al. ${ }^{15}(1997)$, estudando a fauna anofélica que se associa com o homem, em Buriticupu, na Amazônia Maranhense, encontraram 7 espécies: $A n$. argyritarsis, An. oswaldoi, An. nuneztovari, An. rangeli, An. triannulatus davisi, An. darlingi e An. evansae, todas presentes no peridomicílio, enquanto as duas últimas também foram encontradas no intradomicílio. Os mesmos autores mencionaram a presença ainda naquela parte do Estado, de influência amazônica, de mais três espécies, An. albitarsis, An. galvaoi e An. strodei, chamando a atenção para a variedade da fauna, com elementos de padrão de distribuição diverso, sendo algumas espécies típicas de zonas quentes e úmidas, de ambientes amazônicos e outras com ampla distribuição no Brasil.

Na Raposa, também foram encontradas seis espécie e, a exemplo de Buriticupu, todas pertencentes ao subgênero Nyssorhynchus. Entre elas, 4 também ocorrem na Amazônia maranhense: An. albitarsis, An. triannulatus davisi, An. nuneztovari e An. galvaoi. As 3 primeiras foram encontradas em Buriticupu, e a última em Imperatriz (Rebêlo et al. ${ }^{15}, 1997$ ).

Convém destacar dois aspectos importantes do ponto de vista ecológico e epidemiológico nas populações de anofelinos da Raposa: 1) o predomínio na estação chuvosa; e 2) maior atividade hematofágica no crepúsculo vespertino e nas primeiras horas da noite. O primeiro aspecto talvez tenha sido favorecido pelas potencialidades produzidas naquele período, como por exemplo, maior variedade de coleções de água de tamanho e profundidade também variáveis disponíveis para o estabelecimento de criadouros. O segundo deriva da preferência que An. aquasalis e An. albitarsis mostram por aqueles horários (Elliot ${ }^{5}, 1968$; Deane $^{4}$, 1986; Flores-Mendoza ${ }^{6}, 1996$ ), constituindo-se em um aspecto epidemiologicamente importante pois coincide com o horário em que as pessoas, na zona rural, costumam chegar do trabalho ou reunir-se nos arredores da casa, tornando-se mais expostas às investidas dos mosquitos.

O predomínio de An. aquasalis foi favorecido pela proximidade das coleções de água salobra. A localidade de Primirim (Raposa) está situada próximo à faixa litorânea, dominada pelo manguezal, onde há associações de solos alagados, salinos e mal drenados. Como se sabe, este mosquito é o principal vetor da malária na região litorânea do Brasil, pois cria-se em pequenas ou grandes coleções de água com ligeiro teor de salinidade, daí sua distribuição costeira e predomínio no período chuvoso, característica imposta pela relativa transitoriedade de seus criadouros (Consoli e Lourenço-de-Oliveira² ${ }^{2}$ 1994).

An. aquasalis é considerado um anofelino dotado de variável comportamento alimentar quanto à fonte sangüínea (Senior-White ${ }^{16}$, 1952) sendo capaz de sugar, espontaneamente, homem, boi, cavalo, porco, cão e aves, mostrando preferência por animais de grande porte (Flores-Mendoza et al. ${ }^{6}, 1996$ ). Normalmente, é considerado um vetor muito menos importante que An. darlingi, capaz de transmitir a malária somente em locais e ocasi- 
ões em que está em alta densidade, quando tem a oportunidade de atacar o homem em maior número. Neste aspecto, An. aquasalis adquire grande importância na transmissão da malária na região de Raposa.

O An. darlingi não ocorre na ilha de São Luís, domina o interior do Estado, sendo o principal transmissor da malária na Amazônia maranhense, onde tem demonstrado tendência à exofilia não apenas em Buriticupu, como em determinadas áreas da Amazônia brasileira (Deane ${ }^{4}$, 1986; Tadei et al. ${ }^{19}$, 1983; Rebêlo et al. $\left.{ }^{15}, 1997\right)$. Mesmo assim, é o anofelino melhor adaptado ao ambiente humano, o mais antropofílico e de comportamento endófilo mais acentuado (OliveiraFerreira et al. $\left.{ }^{14}, 1992\right)$. Na Ilha de São Luís este mosquito é substituído pelo An. aquasalis.

Depois de An. aquasalis, as espécies mais comuns na Raposa foram An. galvaoi e An. albitarsis. Esta última tem sido tratada como um complexo de espécies crípticas, cujos membros ainda não estão devidamente separados, talvez por esse motivo seja um dos anofelinos relacionados com a transmissão da malária humana que tem maior ecletismo em todos os aspectos de seus hábitos. A sua capacidade de invadir domicílios e sua elevada antropofilia fez com que fosse incriminado como vetor da malária humana. Entretanto, o encontro deste mosquito com o oocisto é raro, indicando tratar-se de um vetor potencial da malária, mas não de grande importância epidemiológica. Na região de Imperatriz, Amazônia Maranhense, é a espécie mais importante, depois de An. darlingi (Sousa ${ }^{18}$, 1995).

An. nuneztovari de um modo geral, no Brasil, é essencialmente zoofílica e crepuscular, sendo bem mais freqüente na floresta do que nos ambientes modificados pelo homem (Correa e Ramos ${ }^{3}$, 1942). Suga o sangue humano, mas o faz com maior avidez em animais. Entretanto, na região de Tucuruí/ Marabá, Amazônia paraense, esta espécie tem sido encontrada em alta densidade, apresentando acentuada antropofilia, podendo até atuar como vetor secundário da malária naquela região (Tadei et al. $\left.{ }^{19}, 1983\right)$. Obviamente este papel é desempenhado com maior eficiência pela população que procura atacar o homem dentro de sua habitação, como ocorre na Venezuela e Colômbia (Kitzmiller et al. ${ }^{9}, 1973$; Conn $\left.{ }^{1}, 1990\right)$. No Maranhão esta espécie tem sido encontrada em densidade moderada em áreas de foco de malária, livre da presença de $A n$. darlingi, como se verifica no município de Pedreiras, zona dos cocais maranhenses (Ministério da Saúde ${ }^{13}$, 1997).

An. evansae é uma espécie de ampla distribuição neotropical, sendo considerada de hábitos essencialmente silvestre e zoofílico. Entretanto, em algumas áreas tem sido encontrada em alta densidade, podendo desempenhar papel vetorial secundário, pois exemplares desta espécie já foram encontrados albergando oocistos no estômago (Correa e Ramos ${ }^{3}$, 1942). No presente estudo, An. evansae foi encontrada em baixíssima densidade e somente no ambiente peridomiciliar, portanto seu papel como vetor secundário é duvidoso. Neste aspecto, outras espécies adquirem maior importância. Por outro lado, em Buriticupu, na Amazônia Maranhense, An. evansae foi a segunda espécie mais abundante tanto no extra como no peridomicílio e também foi encontrada dentro das habitações (Rebêlo et al. $\left.{ }^{15}, 1997\right)$. Naquela região este mosquito pode funcionar como vetor secundário e ter maior importância nas áreas onde o An. darlingi não foi encontrado.

An. triannulatus davisi é uma espécie de distribuição geográfica muito ampla no continente americano. Este anofelino não é vetor primário de malária no Brasil e parece ser pouco suscetível aos Plamodium vivax e $P$. falciparum em condições experimentais, pois pequeno número de esporozoítos consegue alcançar e invadir as suas glândulas salivares (Klein et al. $\left..^{10,11}, 1991\right)$. É uma espécie de hábitos zoofílico e exófilo (Consoli e Lourenço-de-Oliveira ${ }^{2}$, 1994), eventualmente ataca o homem, mas em menor freqüência que a outros animais, como equídeos e bovinos (Galvão e Lane ${ }^{7}$, 1941, Lourençode-Oliveira et al. ${ }^{12}, 1989$ ).

Os dados apresentados no presente trabalho devem ser levados em consideração nos programas de controle feitos pelos órgãos de saúde pública, pois o predomínio e o comportamento de An. aquasalis na Raposa indicam que a malária, naquela área, pode estar sendo transmitida tanto dentro como fora das casas, principalmente no período chuvoso e nas primeiras horas da noite.

\section{AGRADECIMENTOS}

Ao senhor Newton Castro por ter concedido o seu sítio para a realização das coletas dos anofelinos.

\section{REFERÊNCIAS}

1. Conn J. A genetic study of the malaria vector Anopheles nuneztovari from Western Venezuela. J Am Mosquito Cont Assoc 1990;6:400-5.

2. Consoli RAGB, Lourenço-de-Oliveira R. Principais mosquitos de importância sanitária no Brasil. Rio de Janeiro: FIOCRUZ; 1994. 
3. Correa R, Ramos AS. Os anofelinos da região meridional do Estado de São Paulo. Arq Hig Saúde Pública 1942;7:37-57.

4. Deane LM. Malaria vectors in Brazil. Mem Inst Oswaldo Cruz 1986;81:5-14.

5. Elliott R. Studies on man-vector contact in some malarious areas in Colombia. Bull World Health Organ 1968;38:239-53.

6. Flores-Mendoza C, Cunha RA, Rocha DS, Lourençode-Oliveira R. Determinação das fontes alimentares de Anopheles aquasalis (Diptera: Culicidae) no Estado do Rio de Janeiro, Brasil, pelo teste de precipitina. Rev Saúde Pública 1996;30:129-34.

7. Galvão AL, Lane J. Observações sobre alguns anofelinos de Salobra, Mato Grosso (Diptera, Culicidae). Rev Bras Hig 1941;11:10-8.

8. Instituto Brasileiro de Geografia e Estatística. Superintendência de Estudos Geográficos e SócioEconômicos. Atlas do Maranhão. Rio de Janeiro; 1984.

9. Kitzmiller JB, Kreutzer RD, Tallaferro, E. Chromossomal differences in populations of Anopheles nuneztovari. Bull World Helth Organ 1973;48:435-55.

10. Klein TA, Lima JBP, Tada MS. Comparative susceptibility of anopheline mosquitoes to Plasmodium falciparum in Rondônia Brazil. Am J Med Hyg 1991;44:598-603.

11. Klein TA, Lima JBP, Tada MS, Miller R. Comparative susceptibility of anopheline mosquitoes in Rondônia Brazil, to infection by Plasmodium vivax. Am J Med Hyg $1991 ; 5: 463-70$
12. Lourenço-de-Oliveira R, Guimarães ARG, Arlé M, Silva TF, Castro MG, Motta MA, Deane LM. Anopheline species, some of their habits and relation to malaria in endemic areas of Rondônia state, amazon region of Brazil. Mem Inst Oswaldo Cruz 1989;84:501-14.

13. Ministério da Saúde. Fundação Nacional de Saúde. Relatório Anual do Serviço de Epidemiologia. São Luís; 1997.

14. Oliveira-Ferreira J, Lourenço-de-Oliveira R, Deane

LM, Daniel-Ribeiro CT. Feeding preference of Anopheles darlingi in malaria endemic areas of Rondônia state Northwestern Brazil. Mem Inst Oswaldo Cruz 1992;87:601-2.

15. Rebêlo JMR, Silva AR, Ferreira LA, Vieira JA Anopheles (Culicidae, Anophelinae) e a malária em Buriticupu-Santa Luzia, Pré-Amazônia Maranhense. Rev Soc Bras Med Trop 1997;30:107-11.

16. Senior-White RA. Studies on the bionomics of Anopheles aquasalis Curry, 1952. Part III. Ind J Malariol 1952;6:31-72.

17. Silva AR. Perspectiva de uma nova estratégia de controle da malária baseada na sua inserção no Sistema Único de Saúde (SUS). São Luís: Universidade Federal do Maranhão; 1999.

18. Sousa JRP. Contribuição ao estudo epidemiológico da malária no Município de Imperatriz, estado do Maranhão. Imperatriz: Universidade Estadual do Maranhão; 1995.

19. Tadei WP, Mascarenhas BM, Podestá MG. Biologia de anofelinos amazônicos. VIII. Conhecimentos sobre a distribuição de espécies de Anopheles na região de Tucuruí-Marabá (Pará). Acta Amazônica 1983;13:103-40. 